\title{
$H \alpha$ Survey of the Local Volume: Isolated Southern Galaxies
}

\author{
S. S. Kaisin ${ }^{1}$, A. V. Kasparova ${ }^{2}$, A. Yu. Knyazev ${ }^{1,3,4}$, and I. D. Karachentsev ${ }^{1}$ \\ ${ }^{1}$ Special Astrophysical Observatory, Russian Academy of Sciences, Nizhnii Arkhyz, \\ Karachai-Cherkessian Republic, 369167 Russia \\ ${ }^{2}$ Sternberg Astronomical Institute, Universitetskii pr. 13, Moscow, 119992 Russia \\ ${ }^{3}$ European Southern Observatory, Garching, Germany \\ ${ }^{4}$ South African Astronomical Observatory, Cape Town, 7925 South Africa
}

Received June 13, 2006.

\begin{abstract}
We present our $H_{\alpha}$ observations of 11 isolated southern galaxies: SDIG, PGC 51659, E 222010, E 272-025, E 137-018, IC 4662, Sag DIG, IC 5052, IC 5152, UGCA 438, and E 149-003, with distances from 1 to $7 \mathrm{Mpc}$. We have determined the total $H_{\alpha}$ fluxes from these galaxies. The star formation rates in these galaxies range from $10^{-1}$ (IC 4662) to $10^{-4} M_{\odot} \mathrm{yr}^{-1}$ (SDIG) and the gas depletion time at the observed star formation rates lies within the range from $1 / 6$ to 24 Hubble times $H_{0}^{-1}$.

Keywords: galaxies, groups of galaxies, interstellar gas in galaxies, galaxy evolution.
\end{abstract}

\section{INTRODUCTION}

Our views of the star formation in galaxies still remain fragmentary. Until recently, the studies have been focused mainly on the brightest objects. Dwarf systems, particularly those of low surface brightness, remained out of sight, primarily because they were difficult to observe. At the same time, a complete picture cannot be obtained if dwarf galaxies are ignored. Being the elements from which large systems were formed through mergers, they play a key role in the formation and evolution of galaxies. Dwarf galaxies are also interesting in that they are the most commonly encountered type of galaxies in the Universe. Clearly, these objects are easiest to study in the Local Volume.

The most complete sample of the Local Volume is presented in the Catalog of Nearby Galaxies (Karachentsev et al. 2004). This catalog contains 450 galaxies whose distances do not exceed $10 \mathrm{Mpc}$. In recent years, about 30 more hitherto unknown nearby dwarf systems have been discovered. An enormous work has been performed to refine the distances of these galaxies based mainly on the luminosities of their red giant branch stars.

The fact that there are no spiral density waves in dwarf galaxies is important for studying the star formation. Nevertheless, irregular galaxies have star formation rates per unit luminosity that are approximately the same as those of spiral galaxies (Hunter and Gallagher 1986). This paper is a continuation of our previous works (Karachentsev et al. 2005; Kaisin and Karachentsev 2006) on the $H \alpha$ survey of Local Volume galaxies.

\section{OBSERVATIONS AND DATA REDUCTION}

We obtained CCD images in the $H \alpha$ line and in the neighboring continuum for 11 galaxies of the Local Volume. The observations were performed at the $2.2 \mathrm{~m}$ ESO telescope from July 
through August 2004. The images were taken with the Wide Field Imager, which consists of eight CCD arrays with a total field of view of $33^{\prime} \times 34^{\prime}$ and a resolution of $0.238^{\prime \prime}$ per pixel.

The data were obtained with two narrow-band filters. Of these, $H \alpha / 7$ (ESO 856) was centered at $\lambda=6588.27 \AA$ and had a width of $\Delta \lambda=74.31 \AA$. The continuum images were taken with the ESO 858 filter centered at $6655.61 \AA$ with a width of $120.78 \AA$. The typical exposures were 800 and $300 \mathrm{~s}$ in the $H \alpha$ line and the continuum, respectively.

The data were reduced using the standard MIDAS package. Each image was reduced as follows. First, we subtracted a bias from all images and then divided them by fields, which were taken on the same observing night as the galaxy in almost all cases. After automatic cosmic-ray hit removal, the images were corrected for scale distortions. To this end, the field in the immediate neighborhood of each galaxy was fitted by a second-degree polynomial.

We obtained the final $H \alpha$ images by reducing the $H \alpha$ and continuum images to the same scale and by applying corrections for airmass and exposure time. To calibrate the fluxes, we used spectrophotometric standard stars observed on the same nights as the objects. Some of our images exhibit ring-shaped reflexes from bright stars. We removed these artifacts when determining the $H \alpha$ fluxes from the galaxies.

\section{RESULTS}

Table 1 present the main characteristics of the 11 galaxies that we observed. Its columns contain the following: galaxy names (numbers) in known catalogs, equatorial coordinates for the epoch 2000.0, standard angular diameters in arcmin and apparent axial ratios, morphological types in de Vaucouleur's digital code, apparent $B$ magnitudes, and distance to the galaxies in Mpc. References to the original data are given in the catalog by Karachentsev et al. (2004).

Table 1:

\begin{tabular}{|c|c|c|c|c|c|c|c|}
\hline Object & $\begin{array}{c}\text { RA } \\
h \mathrm{~m} \mathrm{~s}\end{array}$ & $\begin{array}{l}\text { DEG } \\
\text { o," }\end{array}$ & $\begin{array}{c}a \\
\operatorname{arcmin}\end{array}$ & $\mathrm{b} / \mathrm{a}$ & Type & $\begin{array}{c}B_{t} \\
m a g\end{array}$ & $\begin{array}{c}\mathrm{D} \\
\mathrm{Mpc}\end{array}$ \\
\hline E349-031, SDIG & $00 \quad 08 \quad 13.3$ & -343442 & 1.1 & 0.82 & 10 & 15.48 & 3.21 \\
\hline PGC51659 & 142803.7 & -461806 & 2.4 & 0.38 & 10 & 16.50 & 3.58 \\
\hline E222-010 & 143503.0 & -492518 & 0.9 & 0.44 & 10 & 16.33 & 5.8 \\
\hline E272-025 & 144325.5 & -444219 & 3.0 & 0.50 & 8 & 14.77 & 5.9 \\
\hline E137-018 & 162059.3 & -602915 & 3.2 & 0.34 & 9 & 12.20 & 6.34 \\
\hline IC4662, E102-14 & 174706.3 & -643825 & 2.8 & 0.57 & 9 & 11.74 & 2.44 \\
\hline SagDIG, E594-4 & 192959.0 & -174041 & 2.9 & 0.72 & 10 & 14.12 & 1.04 \\
\hline E074-15, I5052 & 205206.2 & -691214 & 5.9 & 0.14 & 7 & 11.68 & 6.03 \\
\hline E237-027, I5152 & 220241.9 & -511743 & 5.2 & 0.62 & 10 & 11.06 & 2.07 \\
\hline UGCA438 & 232627.5 & -322326 & 1.5 & 0.80 & 10 & 13.86 & 2.23 \\
\hline E149-003 & 235202.8 & -523439 & 2.2 & 0.18 & 9 & 15.0 & 6.4 \\
\hline
\end{tabular}

Figure 1 shows $H \alpha$ plus continuum (left) and $H \alpha$ minus continuum (right) images of the galaxies from our sample. All images are $800 \times 800$ pixels in size, except for the two most extended galaxies ESO $074-15$ and ESO 237-27 with image sizes of $1300 \times 1300$ pixels. Table 
Table 2:

\begin{tabular}{|c|c|c|c|c|c|c|c|c|}
\hline $\begin{array}{l}\text { Object } \\
(1)\end{array}$ & $\begin{array}{r}M_{B} \\
(2)\end{array}$ & $\begin{array}{l}\mathrm{TI} \\
(3)\end{array}$ & $\begin{array}{r}M_{H I} / L \\
(4)\end{array}$ & $\begin{array}{r}A_{H \alpha} \\
(5)\end{array}$ & $\begin{array}{r}g F_{c} \\
(6)\end{array}$ & $\begin{array}{r}\lg \text { SFR } \\
(7)\end{array}$ & $\begin{array}{r}p_{*} \\
(8)\end{array}$ & $\begin{array}{r}f_{*} \\
(9)\end{array}$ \\
\hline SDIG & -12.10 & -0.8 & 0.55 & 0.03 & $\begin{array}{l}-14.02 \\
\end{array}$ & -3.89 & -0.75 & 1.35 \\
\hline PGC51659 & -11.83 & 0.0 & 6.6 & 0.30 & -13.68 & -3.47 & -0.22 & 1.04 \\
\hline E222-010 & -13.60 & -1.4 & 0.84 & 0.60 & -12.88 & -2.25 & 0.29 & -0.36 \\
\hline E272-025 & -14.77 & -1.5 & 0.21 & 0.37 & -12.80 & -2.15 & -0.08 & -0.60 \\
\hline E137-018 & -18.12 & -1.8 & 0.13 & 0.57 & -11.87 & -1.17 & -0.44 & -0.44 \\
\hline IC 4662 & -15.56 & -0.7 & 0.72 & 0.16 & -10.91 & -1.04 & 0.72 & -0.82 \\
\hline SagDIG & -11.49 & -0.3 & 1.1 & 0.28 & -13.09 & -3.96 & -0.58 & 0.62 \\
\hline IC 5052 & -18.13 & -2.2 & 0.35 & 0.12 & -11.71 & -1.05 & -0.32 & -0.14 \\
\hline IC 5152 & -15.67 & -1.1 & 0.36 & 0.06 & -11.80 & -2.06 & -0.35 & -0.09 \\
\hline UGCA438 & -12.94 & -0.7 & 0.81 & 0.03 & -13.51 & -3.72 & -0.92 & 0.83 \\
\hline E149-003 & -14.09 & -1.7 & 0.89 & 0.03 & -12.90 & -2.19 & 0.15 & -0.20 \\
\hline
\end{tabular}

2 gives some of the calculated integrated parameters for the observed galaxies: (1) galaxy names; (2) their absolute magnitudes corrected for the Galactic extinction as prescribed by Schlegel et al. (1998) and for the internal extinction as described in the Catalog of Nearby Galaxies; (3) the "tidal index" TI introduced in the Catalog of Nearby Galaxies to characterize the galaxy's surroundings (negative $T I$ correspond to field galaxies for which the tidal effect of neighbors is negligible); (4) the ratio of the HI mass to the blue luminosity in solar units from the Catalog of Nearby Galaxies corrected for new distance estimates; (5) Galactic extinction $H \alpha / 7$ line, $A(H \alpha)=2.32 \cdot E(B-V)$, where $E(B-V)$ is the color excess from Schlegel et al. (1998); (6) extinction-corrected logarithm of the $H \alpha$ flux from the galaxy, $F_{c}$ , in erg $\mathrm{cm}^{-2} \mathrm{~s}^{-1}$; and (7) star formation rate in the galaxy on a logarithmic scale defined as $S F R\left(M_{\odot} /\right.$ year $)=1.27 \cdot 10^{9} F_{c}(H \alpha) \cdot D^{2}$, (Gallagher et al. 1984), where the distance to the galaxy is in Mpc. The last two columns give the dimensionless parameters $p_{*}$ and $f_{*}$ (see the next section) that which characterize the global star formation activity of the galaxy in the past and in the future, respectively. Note some of the individual properties of the observed galaxies.

E 349-031 = SDIG (Sculptor Dwarf Irregular Galaxy). This low surface brightness galaxy is located in the region of a sparse group (filament) in Sculptor. Karachentsev et al. (2006a) recently determined its distance from the luminosities of red giant branch (RGB) stars. The nearest significant neighbors of SDIG are NGC 7793 and NGC 253, the "crossing time" with which exceeds the Hubble time $T_{0}=H_{0}^{-1}$. Miller (1996) found no appreciable $H \alpha$ emission from this galaxy. However, our SDIG images show several diffuse emission regions marked by the circles in Fig. 1 .

PGC 51659. An elongated low surface brightness galaxy with a bright star projected onto its southeastern edge. This galaxy is located on the far outskirts of the Cen A group. It is among the ten richest galaxies of the Local Volume in HI abundance per unit luminosity. It exhibits appreciable $H \alpha$ emission only on the northwestern side.

E 222-010. A comet-shaped galaxy at low Galactic latitude $\left(10^{\circ}\right)$. Its distance was estimated from the line-of-sight velocity at the Hubble constant $H_{0}=72 \mathrm{~km} \mathrm{~s}^{-1} \mathrm{Mpc}^{-1}$. The 
brightest emission knots in this galaxy are located at its southern boundary.

E 272-025. The central region of the galaxy is surrounded by an extended diffuse halo. Its distance was estimated from the line-of-sight velocity. Compact emission knots with filaments are seen only in the central part of the galaxy.

E 137-018. An isolated Sm-type galaxy at low Calactic latitude $\left(-7.4^{\circ}\right)$. A bright star is projected near the center. This star produces a ring-shaped reflex in the telescope's optical system. The distance to this galaxy, 6.34 Mpc, was first determined by Karachentsev et al. (2006b).

IC 4662. This bright galaxy with extended sites of star formation is one of the nearest representatives of blue compact galaxies (BCGs). Karachentsev et al. (2006a) resolved it into stars and determined its distance, $2.44 \mathrm{Mpc}$, from RGB stars. Apart from bright compact emission regions, this galaxy exhibits a network of sinuous $H \alpha$ filaments, which could be produced by shocks from supernova explosions. The star formation rate, $9.8 \cdot 10^{-2} M_{\odot} \mathrm{yr}^{-1}$, estimated by Hunter and Elmegreen (2004) agrees well with our data. Hidalgo-Games et al. (2001) pointed out that the compact double object 80 southeast of the center of IC 4662 differs greatly from the main galaxy in oxygen abundance. Being a likely dwarf companion, this object can trigger a starburst in the main body of IC 4662, as is commonly observed in BCGs (Pustilnik et al. 2001).

Sag DIG = E 594-04. This dwarf irregular galaxy in Sagittarius is the nearest object in our sample $(\mathrm{D}=1.04 \mathrm{Mpc})$. It is located immediately outside the zero-velocity sphere with a radius of $0.96 \mathrm{Mpc}$ (Karachentsev and Kashibadze 2006). The tidal index for this relatively isolated galaxy is -0.3 . A single compact emission region lies at the southern edge of Sag DIG. According to Hunter and Elmegreen (2004), its $H \alpha$ flux corresponds to $S F R=$ $1.6 \cdot 10^{-4} M_{\odot} \mathrm{yr}^{-1}$, a value very close to our estimate.

IC 5052 = E 074-15. A bright, isolated edge-on Sd galaxy with many compact HII regions seen in its disk. Seth et al. (2005) recently estimated the distance to IC 5052 from RGB stars to be $6.03 \mathrm{Mpc}$. According to Rossa and Dettmar (2003), the star formation rate for this galaxy inferred from its farinfrared flux is $0.08 M_{\odot} \mathrm{yr}^{-1}$, in good agreement with our estimate.

IC $5152=\mathbf{E}$ 237-27. A bright irregular galaxy at a distance of $2.07 \mathrm{Mpc}$ with many complexes of blue stars and dust spots. Talent (1980) and Webster and Smith (1983) performed spectroscopic studies of IC 5152. The presence of bright field stars makes it difficult to analyze the structure of this galaxy in detail.

UGCA 438. This isolated irregular galaxy is located on the near outskirts of the sparse group in Sculptor. A bright star is projected onto its southern side and produces a ring-shaped reflex in the $H \alpha$ image. Miller (1996) obtained an $H \alpha$ image of UGCA 438, but detected no emission. Our $H \alpha$ image exhibits a single emission region at the southern boundary of the galaxy, the flux from which is given in Table 2. This galaxy was investigated in the HI line with the GMRT Indian radio telescope and its HI distribution was found to be highly asymmetric (Begum 2006).

E 149-003. An isolated dwarf galaxy with an oval central region and an extended envelope (a disk?) of low surface brightness. The distance to the galaxy was estimated from its line-ofsight velocity. In the $H \alpha$ line, the central part of the galaxy looks like a comet with a bright compact knot on the northwestern side. 


\section{DISCUSSION}

As we see from the data presented here, the $H \alpha$ images for the 11 galaxies under discussion differ greatly in structure, although all of them belong to late morphological types and are isolated objects. Their emission-line fluxes and star formation rates also differ by hundreds of times. We used the observed star formation rates in galaxies with distances $D<10 \mathrm{Mpc}$ from the literature (van Zee 2000; Gil de Paz et al. 2003; James et al. 2004; Helmboldt et al. 2004; Hunter and Elmegreen 2004; Karachentsev et al. 2005; Kaisin and Karachentsev 2006). Figure 2 shows the distributions of 154 such galaxies with morphological types $T>0$ in absolute magnitude and star formation rate, $S F R$. As follows from these data, $S F R$ correlates with the galaxy luminosity, so the star formation rate per unit luminosity remains approximately the same for both giant and dwarf galaxies. The 11 galaxies that we observed (marked by the hatched squares) closely follow the general dependence.

To characterize the activity phase of star formation in the galaxies, we calculated the following two quantities for them:

$$
\begin{aligned}
& p_{*}=\lg \left\{[S F R] \cdot T_{0} / L_{B}\right\} \text { and } \\
& f_{*}=\lg \left\{M(H I) /[S F R] \cdot T_{0}\right\},
\end{aligned}
$$

where $T_{0}$ is the age of the Universe, which is assumed to be $13.7 \mathrm{Gyr}$ (Spergel et al. 2003), and $M(H I)$ is the total HI mass in the galaxy. The first parameter shows what fraction of the galaxy's observed luminosity it would "gain" in the Hubble time at a typical mass-tolight ratio of $1 M_{\odot} / L_{\odot}$ and the current star formation rate. The second parameter shows how many Hubble times it would take for the galaxy to run out of its gas at the current star formation rate. In fact, both dimensionless parameters, $p_{*}$ and $f_{*}$, indirectly characterize the past and future of the star formation in the galaxy. The last two columns of Table 2 give the parameters $p_{*}$ and $f_{*}$ for the 11 observed galaxies. The solid straight line in Fig. 2 indicates the $p_{*}=0$ line. The dash-dotted lines parallel to it correspond to $p_{*}=+1$ and $p_{*}=-1$. As we see, the $p_{*}=0$ line is close to the regression line for the entire set of spiral and irregular galaxies in the Local Volume.

The star formation in a galaxy can follow various scenarios: (a) gradual depletion of the initial reserves of gas in the regime of autonomous steady "smoldering"; (b) semiautonomous evolution without any in flow/outflow of gas, but in the regime of starbursts triggered by external factors (tides); (c) nonautonomous evolution with a significant inflow of gas from the intergalactic medium or with the sweepout of gas from a dwarf galaxy as it passes through the periphery of a giant galaxy; (d) evolution driven by frequent galaxy mergers. Some of these scenarios were considered by Pustilnik et al. (2004), Tutukov (2006a, 2006b), and other authors. We assume that the distribution of galaxies in the $\left\{p_{*}, f_{*}\right\}$ diagram can suggest what type of evolution agrees better with the available observational data. Care should be taken that the sample of galaxies is limited by a well-defined criterion (fixed distance, flux, and/or morphological type). For example, our small sample of isolated galaxies shows an anticorrelation between the parameters $\left\{p_{*}\right.$ and $\left.f_{*}\right\}$ with a coefficient of 0.72 (see Fig. 3). This may indicate that the starburst scenario in isolated galaxies or in galaxies located in the relatively poor surroundings of neighboring galaxies predominates. We emphasize that the measurement errors of $\log S F R$ are small $( \pm 0.08)$ and cannot be responsible for the spread of galaxies along the indicated diagonal line by an order of magnitude.

\section{Aknowledgements}


We wish to thank A.V. Tutukov for helpful advice. This work was supported in part by the Russian Foundation for Basic Research (project no. 04-02-16115).

\section{REFERENCES}

1. A. Begum, private communication (2006).

2. J. S. Gallagher, D. A. Hunter, and A. V. Tutukov, Astrophys. J. 284, 544 (1984).

3. A. Gil de Paz, B. F. Madore, and O. Pevunova, Astrophys. J., Suppl. Ser. 147, 29 (2003).

4. J. F. Helmboldt, R. A. Walterbos, G. D. Bothun, et al., Astrophys. J. 613, 914 (2004).

5. A. M. Hidalgo-Games, J. Masegosa, and K. Olofsson, Astron. Astrophys. 369, 797 (2001).

6. D. A. Hunter and J. S. Gallagher, Publ. Astron. Soc. Pac. 98, 5 (1986).

7. D. A. Hunter and B. G. Elmegreen, Astron. J. 128, 2170 (2004).

8. P. A. James, N. S. Shane, J. E. Beckman, et al., Astron. Astrophys. 414, 23 (2004).

9. S. S. Kaisin and I. D. Karachentsev, submitted to Astrofizika.

10. I. D. Karachentsev and O. G. Kashibadze, Astrofizika 49, 5 (2006) [Astrophys. 49, 3 (2006)].

11. I. D. Karachentsev, V. E. Karachentseva, W. K. Huchtmeier, and D. I. Makarov, Astron. J. 127, 2031 (2004).

12. I. D. Karachentsev, S. S. Kajsin, Z. Tsvetanov, and H. Ford, Astron. Astrophys. 434, 935 (2005).

13. I. D. Karachentsev, A. Dolphin, R. B. Tully, et al., Astron. J. 131, 1361 (2006a).

14. I. D. Karachentsev, R. B. Tully, A. Dolphin, et al., Astron. J., submitted (2006b).

15. B. W. Miller, Astron. J. 112, 991 (1996).

16. S. A. Pustilnik, A. Y. Kniazev, V. A. Lipovetsky, and A. V. Ugrymov, Astron. Astrophys. 373, 24 (2001).

17. S. A. Pustilnik, A. G. Pramskij, and A. Y. Kniazev, Astron. Astrophys. 425, 51 (2004).

18. J. Rossa and R.-J. Dettmar, Astron. Astrophys. 406, 505 (2003).

19. A. C. Seth, J. J. Dalcanton, and R. C. de Jong, Astron. J. 129, 1331 (2005).

20. D. J. Schlegel, D. P. Finkbeiner, and M. Davis, Astrophys. J. 500, 525 (1998).

21. D. N. Spergel, L. Verde, H. V. Peiris, et al., Astrophys. J., Suppl. Ser. 148, 175 (2003).

22. D. L. Talent, PhD Thesis (Rice Univer., Houston, 1980).

23. A. V. Tutukov, Pis'ma Astron. Zh. (in press).

24. A. V. Tutukov, Astron. Rep. 50, 439 (2006).

25. L. van Zee, Astron. J. 119, 2757 (2000).

26. B. L. Webster and M. G. Smith, Mon. Not. R. Astron. Soc. 204, 743 (1983). 

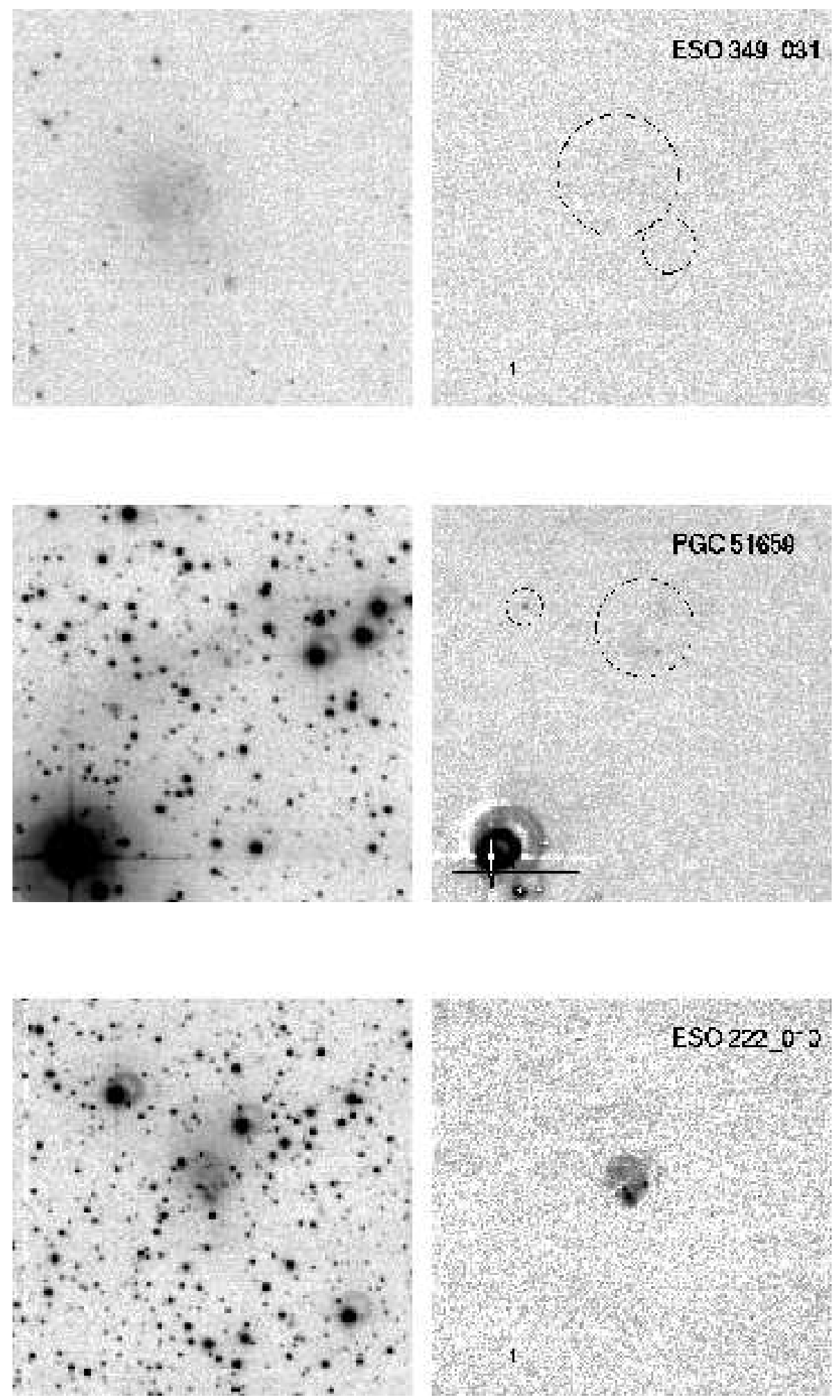

Figure 1: Images of the observed southern galaxies: $H \alpha+$ continuum (left) and $H \alpha$ - continuum (right). 

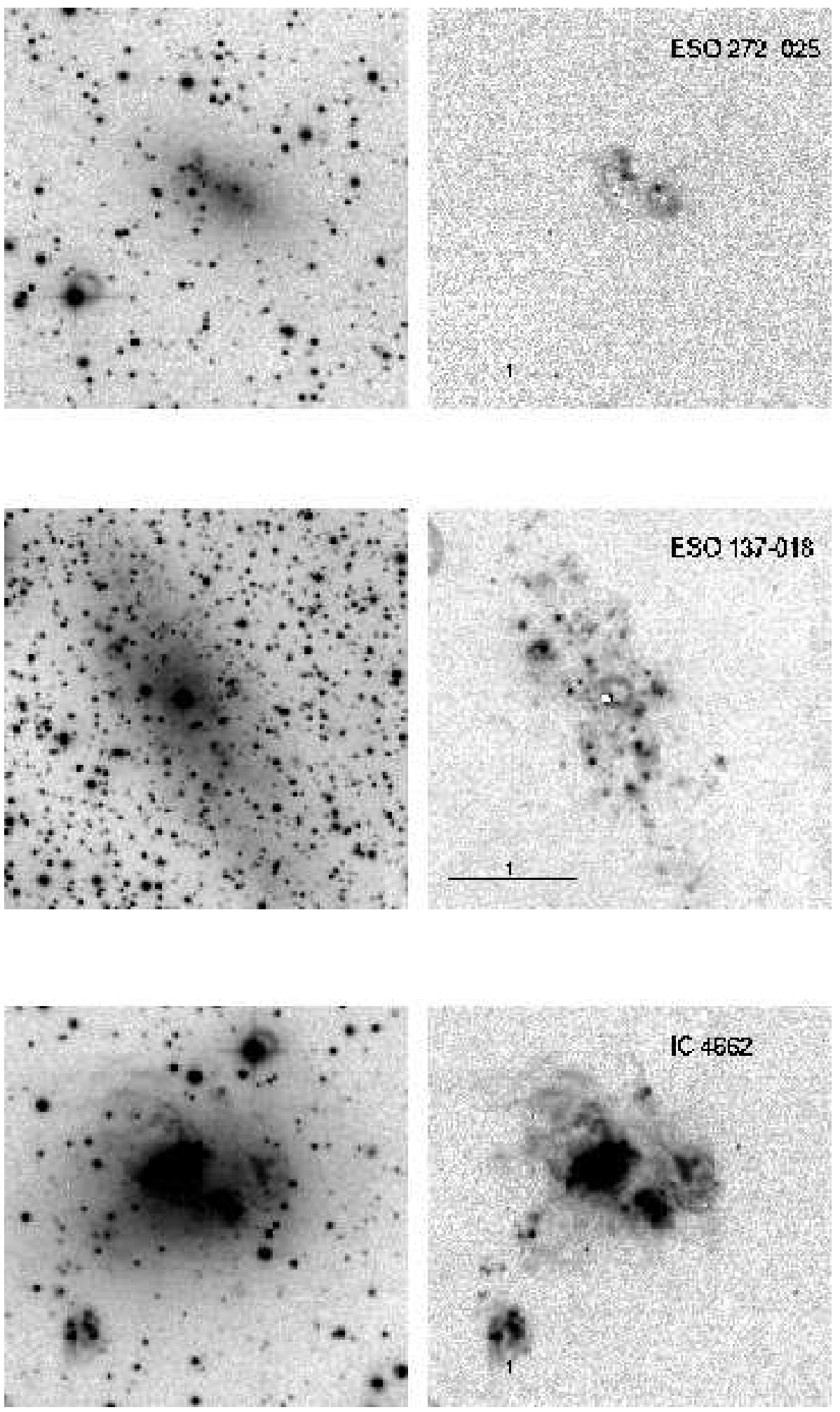

Figure 1: Continued 

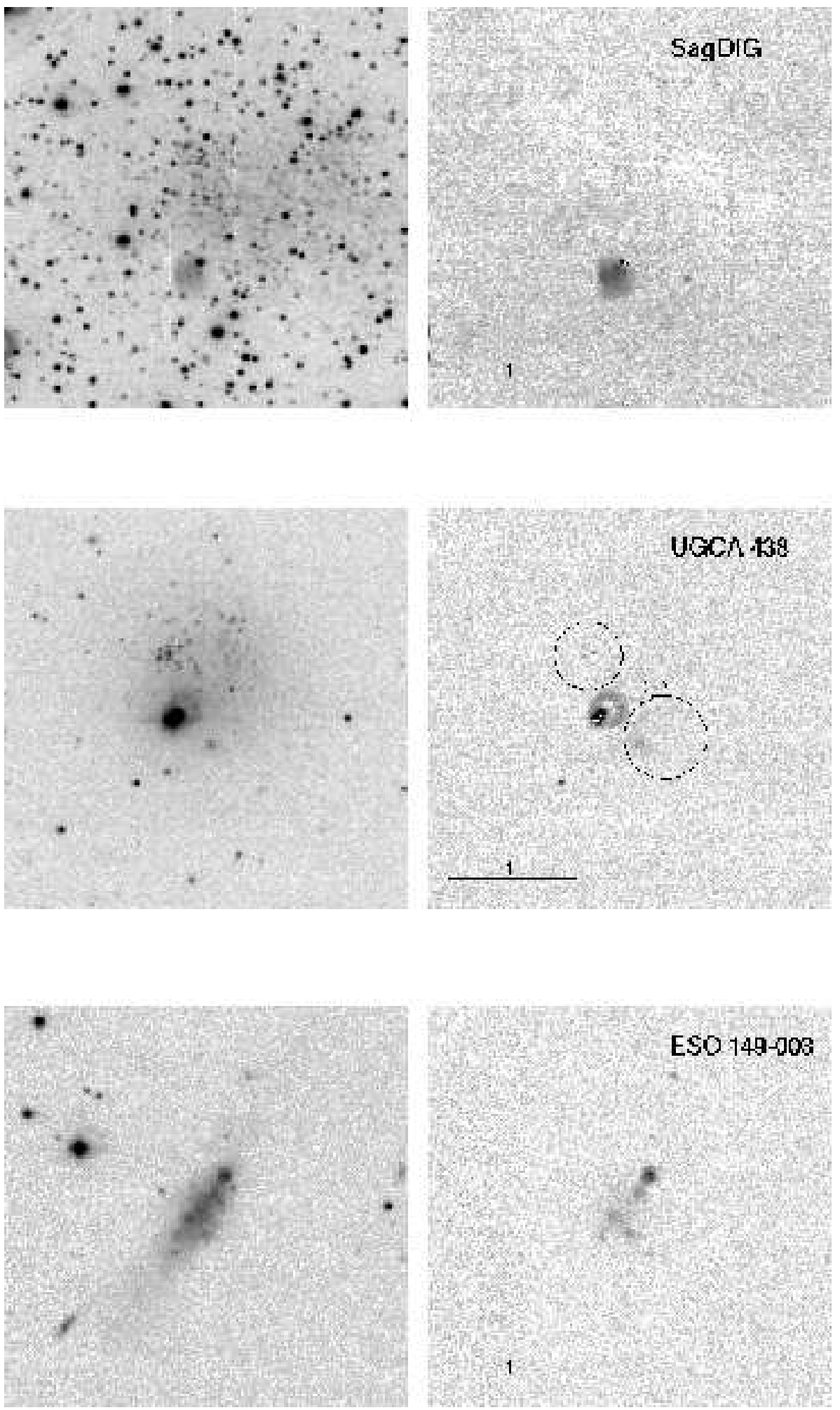

Figure 1: Continued 

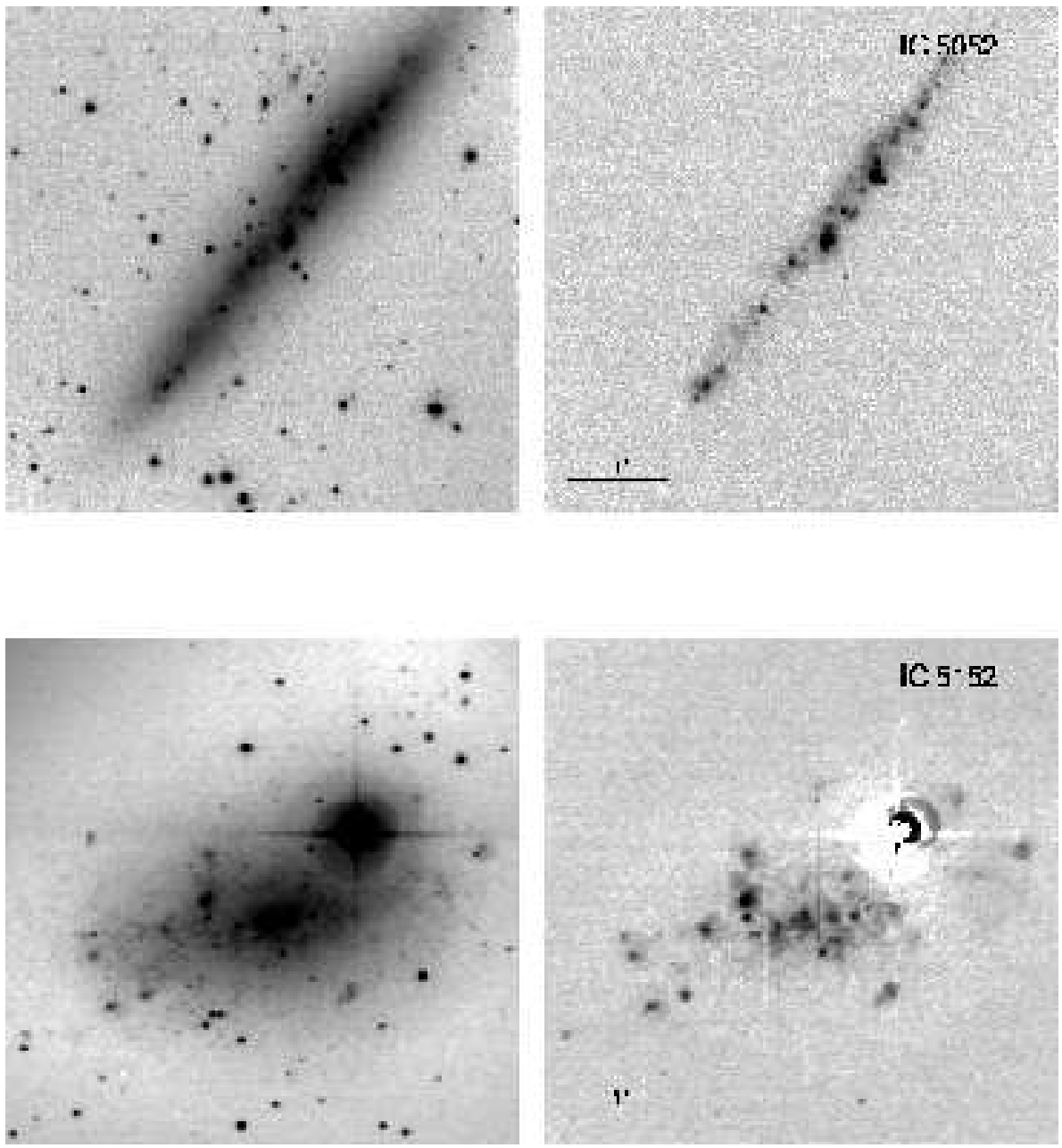

Figure 1: Continued 


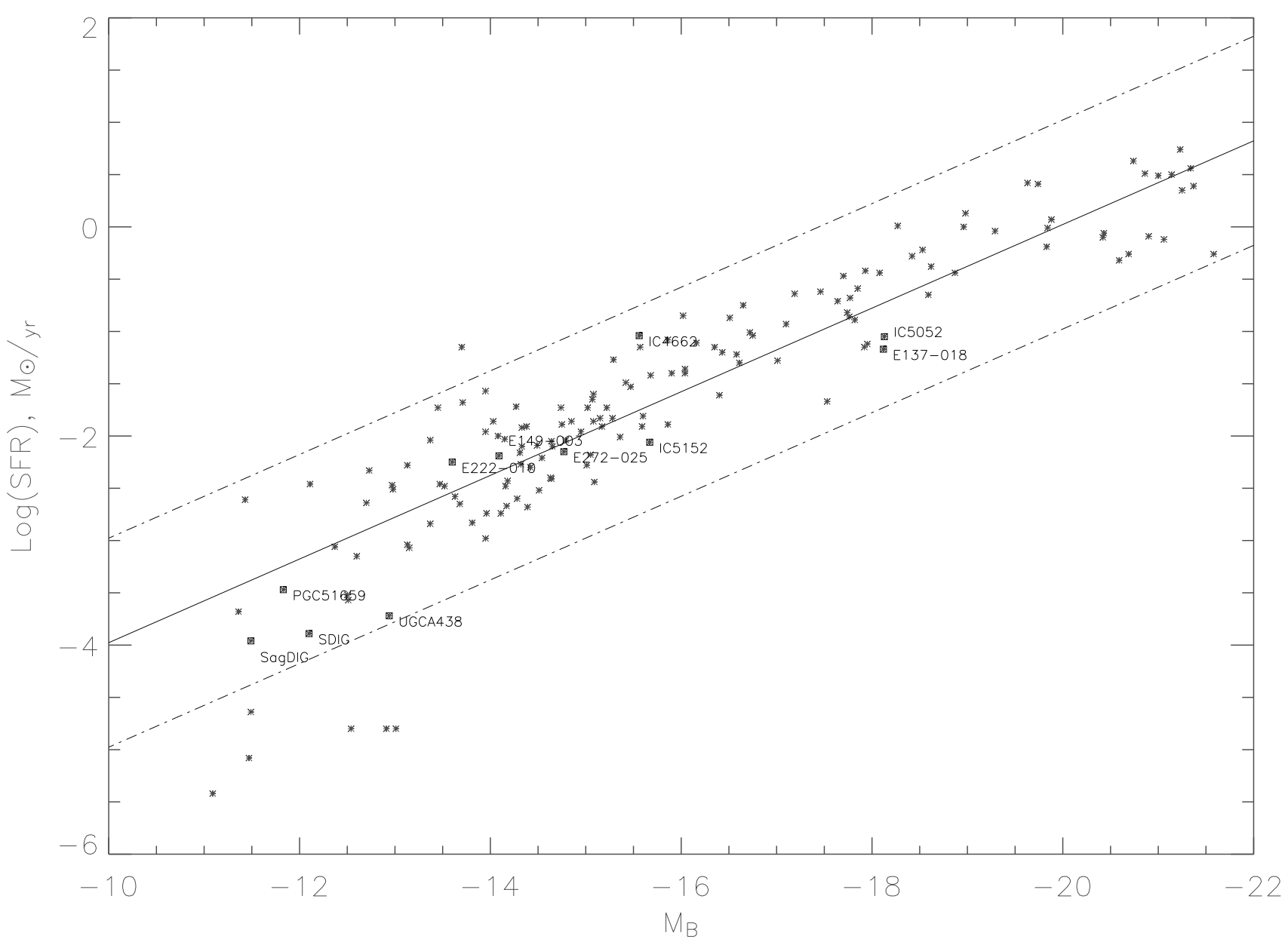

Figure 2: Distribution of the Local Volume galaxies in current star formation rate and absolute magnitude. 


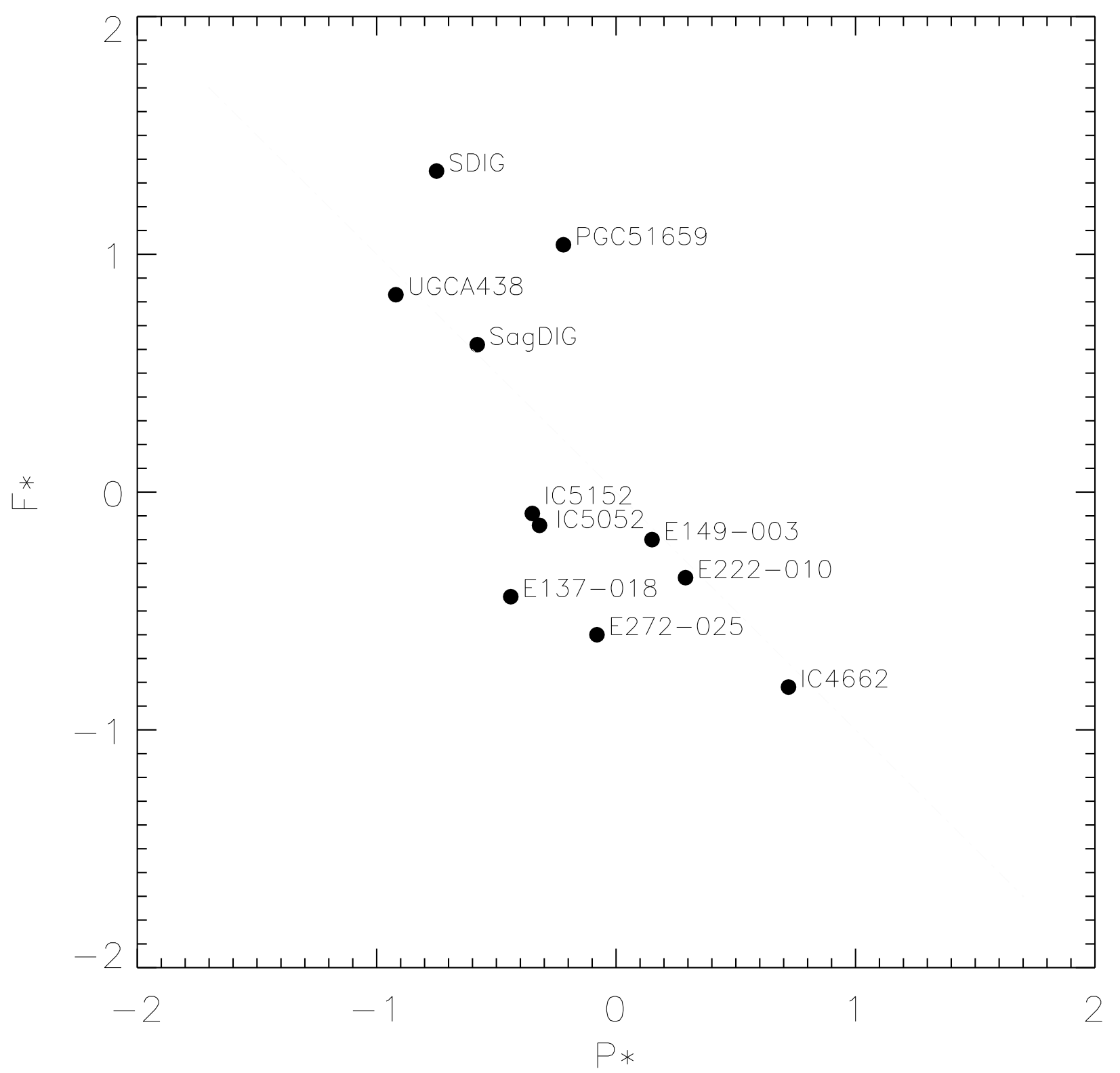

Figure 3: Distribution of the observed galaxies in $p_{*}$ and $f_{*}$ 